\title{
Bacteriological Study of Neonatal Septicemia and their Antibiotic Susceptibility Patterns at MGM Hospital, Warangal, India
}

\author{
Ravindar Sirikonda and Kondal Rao Ravilla*
}

Department of Microbiology, Kakatiya Medical College, Warangal, Telangana, India

*Corresponding author

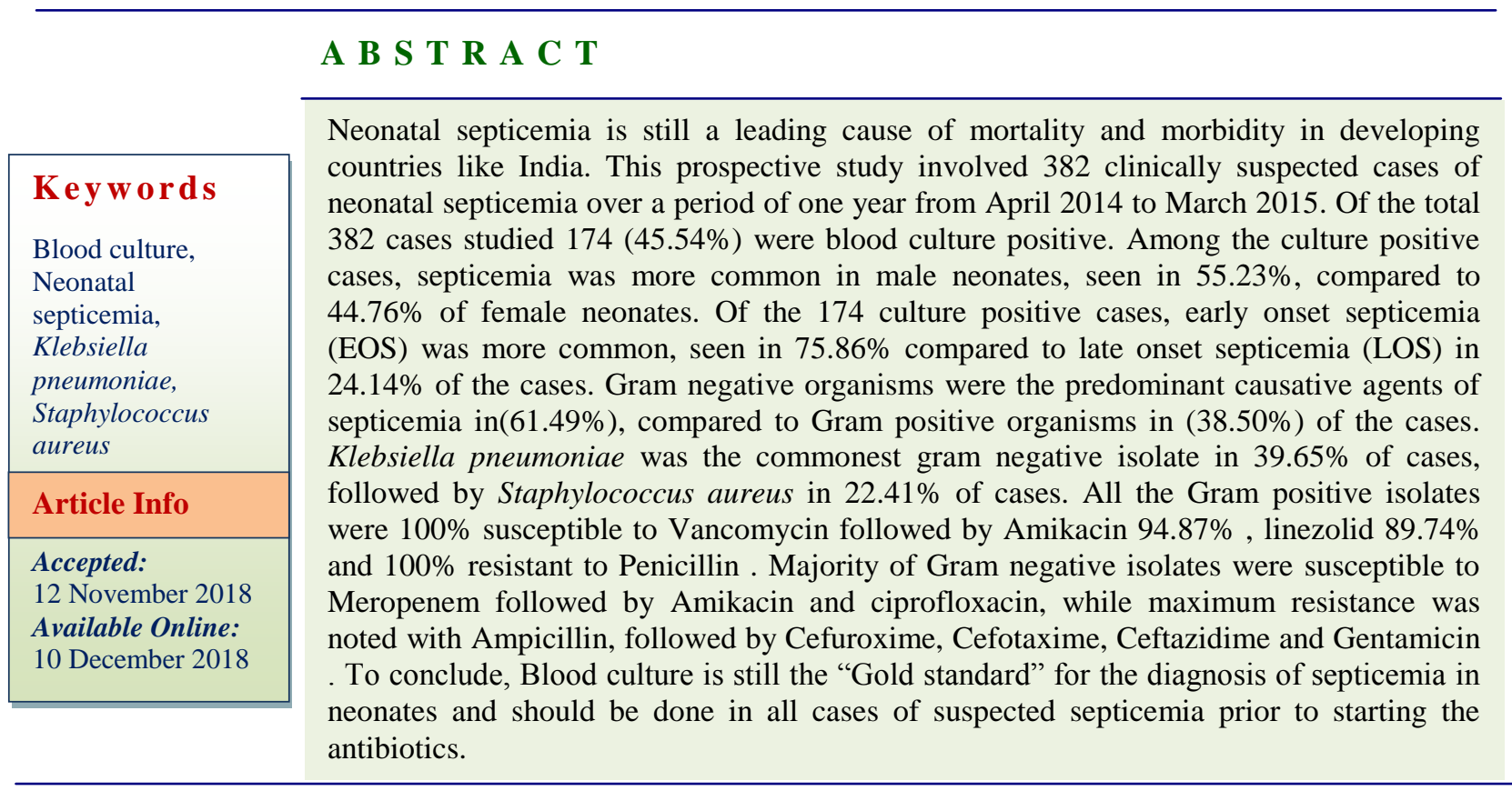

\section{Introduction}

Sepsis is the commonest cause of neonatal mortality; and is the four leading causes of neonatal mortality and morbidity in India ${ }^{1}$. It is estimated that up to $20 \%$ of neonates develop sepsis and approximately $1 \%$ die of sepsis related causes ${ }^{2}$. The risk factors for Neonatal septicemia include premature rupture of membranes, prolonged rupture of membranes, prematurity, UTI, poor maternal nutrition, LBW, birth asphyxia and congenital anomalies ${ }^{3}$.

Mortality rate in neonatal sepsis differs according to the type of organism involved. Gram negative bacteria caused the highest mortality. Among the intramural births, Klebsiella pneumoniae was the most frequently isolated followed by Staphylococcus aureus. 
Among the extramural neonates (referred from community) Klebsiella pneumoniae was again the commonest organism isolated followed by Staphylococcus aureus and Pseudomonas ${ }^{4}$. A study by Shrestha and co-workers observed that Klebsiella is the most common organism which is almost resistant to first line antibiotics ${ }^{5}$. Whereas, Gram negative and Gram positive isolates are resistant to first line antibiotics and even with Cefotoxim is emerging and is a major concern.

The successful treatment with a favorable outcome of the neonate depends on an ongoing review of the causative organisms and their antibiotic susceptibility pattern ${ }^{6}$. Bacterial organisms with increased antibiotic resistance have also emerged and have further complicated the management of neonatal sepsis ${ }^{7}$.

Several studies have shown the resistance pattern exhibited by the organisms isolated from sepsis patients, Gyawali and co-workers observed high incidence of neonatal septicemia and organisms isolated exhibited higher resistance towards commonly used antimicrobials ${ }^{8}$.

It is important that supportive and antimicrobial therapy of a neonate with sepsis is instituted quickly. Hence only a minimum of essential investigations should be undertaken. Blood culture is used as gold standard for diagnosis of septicemia and would be performed in all cases of suspected sepsis prior to starting antibiotics4. In the recent past, a study by Jyothi and co-workers concluded that, blood culture from all neonates suspected to have septicemia showed Gram negative organisms (Klebsiella, Acinetobacter), CONS and S. aureus are the leading cause of neonatal sepsis and also observed that most of them are resistant to multiple antibiotics ${ }^{9}$. The present study was aimed to study the Bacteriological profile and antibiotic susceptibility pattern of neonatal septicemia at MGM Hospital, Warangal.

\section{Materials and Methods}

Source of the data: This prospective study was conducted in the Department of Microbiology, Mahatma Gandhi Memorial Hospital, Warangal a period of 1year from April 2014 to March 2015. Blood samples from 382 clinically suspected neonatal septicemia cases are subjected to aerobic culture. The culture isolates obtained are tested for antibiotic susceptibility pattern.

Inclusion criteria: Neonates were included when at least three of the following risk factors were present ${ }^{9}:$ 1) Febrile illness in the mother during or within two weeks of delivery (more than $38^{0} \mathrm{C}$, oral temperature). 2) More than 3 vaginal examinations during labor. 3) Prolonged rupture of membranes (more than 12 hours). 4) Foul smelling or meconium stained liquor. 5) Preterm baby or LBW baby. 6) Birth asphyxia and difficult resuscitation. 7) Pathological evidences of funisitis.

In addition, neonates who presented with symptoms of septicemia like refusal of feeds, decreased activity, lethargy, respiratory distress, fever, hypothermia, sclerema, abdominal distension, seizures and shock were all included.

Exclusion criteria: 1) Age>28days, 2) Neonates with lethal congenital anomalies.

\section{Sample collection}

An area of approximately $5 \mathrm{~cm}$ over the vein puncture site was disinfected with 70\% alcohol gently and allowed to dry. This was followed by application of Povidone Iodine in concentric circles over the site and allowed to dry for at least 1 minute. About $1 \mathrm{ml}$ of blood was drawn using a sterile syringe and 
inoculated aseptically into a blood culture bottle (brain heart infusion broth).

\section{Blood culture}

About $1 \mathrm{ml}$ of blood will be drawn aseptically and inoculated into a blood culture bottle containing $10 \mathrm{ml}$ of Brain Heart Infusion broth, thus making a dilution of 1 in 10 to nullify the natural bacteriostatic/bactericidal activity of blood. Brain Heart Infusion broth will be prepared using the commercially available ready to use powder, supplied by Himedia Laboratories Pvt. Limited. The broth will be distributed into $10 \mathrm{ml}$ quantity in McCartney bottles and sterilized by autoclaving at $121^{\circ} \mathrm{C}$ for 15 minutes. After inoculation, the blood culture bottles will be incubated at $37^{\circ} \mathrm{C}$ under aerobic conditions in the incubator for 7 days. The first subculture will be done after 24 hours of incubation, the second on the third day and a final on the seventh day. Subcultures will be done onto 5\% sheep blood agar and Mac Conkey agar plates. The inoculated plates will be incubated aerobically in the incubator at $37^{\circ} \mathrm{C}$ for 24hours, and the plates were observed for growth. [AIIMS -NICU protocol 2008]. The growth will be identified by colony characteristics, Gram's stain and standard biochemical tests described in Mackie and McCartney, Practical Medical Microbiology ${ }^{10}$, and Bailey and Scott's Diagnostic Microbiology ${ }^{11}$. Cultures which did not yield any growth following three subcultures were reported negative at the end of 7 days.

\section{Antibiotic susceptibility testing}

Antibiotic susceptibility testing will be done for all the isolates on Muller Hinton agar using commercially available discs (Hi media), by Kirby- Bauer disc diffusion technique as per the CLSI guidelines. Two or three identical colonies were taken from the primary culture plates with sterile loop and suspended in BHI broth, incubated at $37^{\circ} \mathrm{C}$ The turbidity was compared and adjusted to $0.5 \mathrm{Mc}$ Farland tube turbidity standards.

A sterile swab was dipped into the inoculum. Excess inoculum was removed by pressing and rotating the swab firmly against the side of the tube, above the level of liquid. The swab was streaked all over the surface of a Muller Hinton Agar plate. Finally swab was passed round the edge of the agar surface. The inoculum was dried for a few minutes at room temperature with the lid closed. Commercially available antibiotic discs obtained from HiMedia laboratories Ltd, were used. The antibiotic discs were placed on the inoculated plates using a pair of sterile forceps and gently pressed to ensure even contact. The plates were incubated at $37^{\circ} \mathrm{C}$ After 16-18hrs incubation the diameter of each zone was measured with a scale, recorded in $\mathrm{mm}$ and interpreted as sensitive or resistant, in accordance to the indications of the disc manufacturer. The results were recorded and interpreted as per CLSI recommendations.

\section{Results and Discussion}

In the present study, out of the total 382 clinically suspected neonates, 174 (44.54\%) blood culture positive and $208(54.45 \%)$ blood culture negative (Table 1). Out of 382 cases of clinical sepsis $211(55.23 \%)$ were male neonates, $171(44.76 \%)$ were female neonates. Male neonates with clinical sepsis were admitted more frequently than female neonates, which is statistically significant. (pvalue 0.04 ). Among 174 cases of proven sepsis $91(52.29 \%)$ were male neonates and 83 $(47.7 \%)$ were females neonates. There was no sex difference in blood culture positive sepsis (p-value 0.54) (Table 2).

In the cohort of neonates, with clinical sepsis term neonates were more than 215 (56.28\%). pre term neonates 167 (43.71\%) which is 
statistically significant (0.01). However, Blood culture positivity was comparable in both preterm 106(60.91\%) and term 68 $(39.08 \%)$ neonates (p-value 0.003) (Table 3).

Of the total 174 culture positive cases, Early onset septicemia was seen in $75.86 \%$ cases and $24.14 \%$ cases showed late onset septicemia, indicating majority of culture positive cases were seen among early onset septicemia (Figure 1).

Out of 382 clinical sepsis 174 (45.54\%) were blood culture positive. Out of 174 organisms isolated in blood culture, 67(38.5\%) were gram positive and 107(61.49\%) were gram negative (Table 4).

In the present study, Klebsiella species (39.65\%) was the most common organism in our study followed by Coagulase positive Staphylococcus $(22.41 \%)$ and CONS (16.09\%). In gram positive organisms most common are coagulase positive Staphylococcus (22.41\%), CONS (16.09\%). In Gram negative organisms most common are Klebsiella species (39.65\%), followed by
E. coli (14.94\%) and Pseudomonas (6.89\%) (Table 5).

In the present study majority of isolates were Gram negative accounting for $61.49 \%$ of the total cases, commonest organism was Klebsiella pneumoniae susceptible to Meropenem (91.3\%), Amikacin (84.05\%), Ciprofloxacin (56.52\%), Ceftazidime $(31.81 \%)$ and Gentamicin (31.81\%). Most of the isolates were resistant to Ampicillin and Cefuroxime. In Gram positive organisms commonest was S.aureus $(22.41 \%), 100 \%$ sensitive to Vancomycin followed by Amikacin (94.87\%), Linezolid (89.74\%) and Ciprofloxacin $(87.17 \%)$. E. coli isolates were susceptible to Meropenem (88.46 \%), followed by Amikacin (84.05\%). Majority were resistant to Ampicillin, cefuroxime, Cefotaxime and Gentamicin. Other less commonly isolated Gram negative organisms were $P$. aeruginosa $(6.89 \%)$, was sensitive to Meropenem $91.66 \%$ followed by Amikacin $83.33 \%$ and Ciprofloxacin 25\%, and 100\% resistant to Ampicillin and Cefuroxime (Table $6)$.

Table.1 Blood culture positive cases among total cases

\begin{tabular}{|l|l|l|}
\hline Blood Culture & Number of Patients & Percentage \\
\hline Positive & 174 & 45.54 \\
\hline Negative & 208 & 54.45 \\
\hline & 382 & 100 \\
\hline
\end{tabular}

Table. 2 Sex distribution among clinical and blood culture positive sepsis

\begin{tabular}{|c|c|c|c|c|}
\hline SEX & $\begin{array}{l}\text { CLINICAL } \\
\text { SEPSIS }\end{array}$ & & $\begin{array}{l}\text { BLOOD } \\
\text { CULTURE } \\
\text { POSITIVE } \\
\text { SEPSIS }\end{array}$ & \\
\hline Male & $211(55.23 \%)$ & & $91(52.29 \%)$ & \\
\hline Female & $171(44.76 \%)$ & & $83(47.7 \%)$ & \\
\hline Total & 382 & $\begin{array}{l}\text { p-value } \\
0.04\end{array}$ & 174 & $\begin{array}{l}\mathrm{p} \text { - value } \\
0.54\end{array}$ \\
\hline
\end{tabular}


Table.3 Gestational age distribution among clinical and blood culture positive sepsis

\begin{tabular}{|c|c|c|c|c|}
\hline $\begin{array}{l}\text { GESTATION } \\
\text { AGE }\end{array}$ & $\begin{array}{l}\text { CLINICAL } \\
\text { SEPSIS }\end{array}$ & & $\begin{array}{l}\text { BLOOD } \\
\text { CULTURE } \\
\text { POSITIVE } \\
\text { SEPSIS }\end{array}$ & \\
\hline Preterm & $167(43.71 \%)$ & & $106(60.91 \%)$ & \\
\hline Term & $215(56.28 \%)$ & & $68(39.08 \%)$ & \\
\hline Total & 382 & $\begin{array}{l}\text { p-value } \\
0.01\end{array}$ & 174 & $\begin{array}{l}\text { p-value } \\
0.003\end{array}$ \\
\hline
\end{tabular}

Table.4 Bacterial profile in blood culture positive sepsis

\begin{tabular}{|l|l|l|l|}
\hline & $\begin{array}{l}\text { Gram positive cocci } \\
\text { Blood culture sepsis }\end{array}$ & $\begin{array}{l}\text { Gram negative bacilli } \\
\text { Blood culture sepsis }\end{array}$ & $\begin{array}{l}\text { Bacterial } \\
\text { Sepsis (total) }\end{array}$ \\
\hline No. of organisms & $67(38.50 \%)$ & $107(61.49 \%)$ & 174 \\
\hline
\end{tabular}

Table.5 Spectrum of isolates in blood culture positive sepsis

\begin{tabular}{|l|l|}
\hline Organism & No of Patients \\
\hline Klebsiella species & $69(39.65 \%)$ \\
\hline E. coli & $26(14.94)$ \\
\hline Pseudomonas & $12(6.89 \%)$ \\
\hline Coagulase positive Staphylococcus & $39(22.41 \%)$ \\
\hline CONS & $28(16.09 \%)$ \\
\hline TOTAL & 174 \\
\hline
\end{tabular}

Table.6 Antibiotic susceptibility pattern of the isolates

\begin{tabular}{|c|c|c|c|c|c|c|c|c|c|c|}
\hline \multirow[t]{2}{*}{ ANTIBIOTICS } & \multicolumn{2}{|c|}{ К. рпеи (69) } & \multicolumn{2}{|c|}{ E. coli (26) } & \multicolumn{4}{|c|}{ P. aerug (12) S. aureus (39) } & \multicolumn{2}{|c|}{ CONS(28) } \\
\hline & S\% & $\mathbf{R} \%$ & S\% & $\mathbf{R} \%$ & S\% & $\mathbf{R} \%$ & S\% & $\mathbf{R \%}$ & S\% & $\mathbf{R} \%$ \\
\hline PENICILLIN (P) & - & - & - & - & - & - & 0 & 100 & 7.14 & 92.85 \\
\hline ERYTHROMYCIN (E) & - & - & - & - & - & - & 5.12 & 94.87 & 17.85 & 82.14 \\
\hline VANCOMYCIN (VA) & - & - & - & - & - & - & 100 & 0 & 100 & 0 \\
\hline LINEZOLID (LZ) & - & - & - & 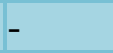 & - & - & 89.74 & 10.25 & 92.85 & 7.14 \\
\hline AMPICILLIN (AMP) & 5.79 & 94.20 & 19.23 & 80.79 & 0 & 100 & 2.56 & 97.43 & 14.28 & 85.71 \\
\hline GENTAMICIN (G) & 31.88 & 68.11 & 53.84 & 46.15 & 8.33 & 91.66 & 82.05 & 17.94 & 89.28 & 10.71 \\
\hline AMIKACIN (AK) & 84.05 & 15.94 & 84.61 & 15.38 & 83.33 & 16.66 & 94.87 & 5.12 & 96.42 & 3.57 \\
\hline CIPROFLOXACIN (CIP) & 56.52 & 43.47 & 30.76 & 69.23 & 25 & 75 & 87.17 & 12.82 & 82.14 & 17.85 \\
\hline CEFOTAXIME(CTX) & 23.18 & 76.81 & 26.92 & 73.07 & 8.33 & 91.66 & 69.23 & 30.76 & 75 & 25 \\
\hline CEFUROXIME (CXM) & 13.04 & 86.95 & 23.07 & 76.92 & 0 & 100 & 12.82 & 87.17 & 39.28 & 60.71 \\
\hline CEFTAZIDIME(CA) & 31.88 & 68.18 & 30.76 & 69.23 & 16.66 & 83.33 & 71.79 & 28.2 & 89.28 & 10.71 \\
\hline MEROPENEM(MRP) & 91.30 & 8.69 & 88.46 & 11.53 & 91.66 & 8.33 & & - & & - \\
\hline
\end{tabular}

K.pneu - Klebsiella pneumoniae; E. coli - Escherichia coli; P. aerg - Pseudomonas aeruginosa; S. aureus Staphylococcus aureus; CoNS - Coagulase Negative Staphylococci 
Figure.1 shows schematic distribution of culture positive cases according to onset of symptoms $(\%)$

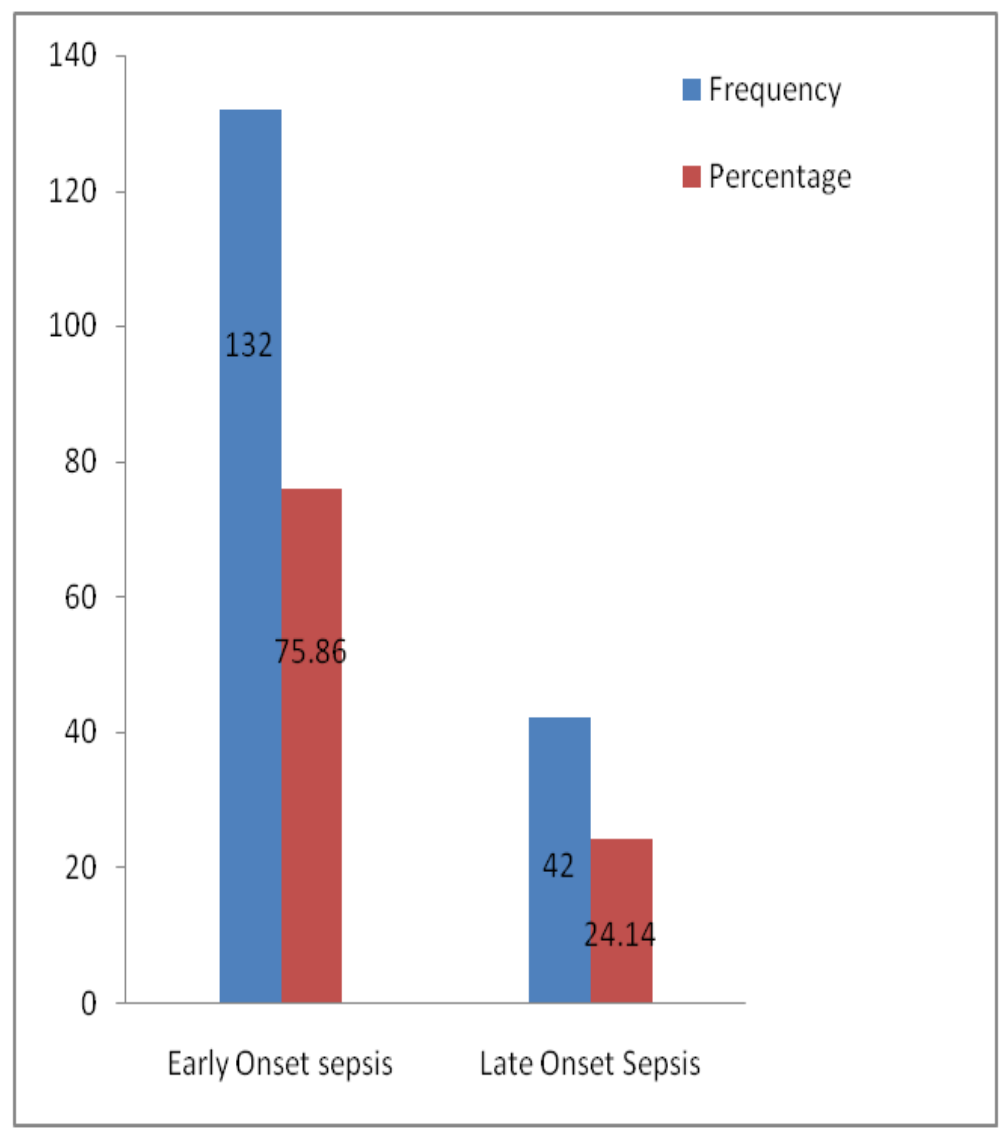

Bacterial septicemia is still a major cause of morbidity and mortality in the newborn period, particularly in the developing countries despite the considerable progress in early diagnosis and treatment measures ${ }^{12}$. The disease continues to pose a challenge to the pediatricians in making a definitive clinical diagnosis due to the subtle and nonspecific signs and symptoms; hence laboratory diagnosis plays a major role. Definitive diagnosis rests on a positive blood culture, to identify the pathogen and determine its antibiotic susceptibility pattern, but for better survival and outcome, simple and rapid diagnostics tests are required as adjuncts to the blood culture for early and effective initiation of treatment to the septicemic neonates.
In the present study an attempt has been made to know the various etiological agents responsible for neonatal septicemia and their antibiotic susceptibility patterns.

This prospective study involved 382 clinically suspected cases of neonatal septicemia. Of the total 382 cases studied 174 (45.54\%) were blood culture positive. Among the culture positive cases, septicemia was more common in male neonates, compared to female neonates. Similar observations are seen by earlier studies ${ }^{13}$. In the present study culture positive septicemia cases were higher among the preterm neonates. The results of our study were comparable to the studies conducted by Bhat and co-workers ${ }^{14}$. Higher number of cases in preterm neonates may be due to 
predisposing factors. Of the 174 culture positive cases, early onset septicemia was more common, compared to late onset septicemia. The higher number of EOS cases may be due to the immature immunological responses of the neonates in the first week of life, making them more susceptible to infections in this period ${ }^{15,18}$. Present study also shows that, culture positive septicemia cases were higher among males than the females, which is in accordance with earlier studies ${ }^{16}$.

Male preponderance in neonatal septicemia may be linked to the $\mathrm{X}$ - linked immunoregulatory gene factor resulting in the host's susceptibility to infections in males. In the present study, Klebsiella pneumoniae was the predominant isolate followed by $S$. aureus. Gram negative organisms were more compared to Gram positive organisms causing septicemia, present observations were in accordance with earlier studies ${ }^{17}$. In the present study, Staphylococcus aureus was the commonest isolate in Gram positive organisms, $100 \%$ sensitive to Vancomycin, $100 \%$ resistant to Penicillin. But study conducted by Neema Kayange and coworkers ${ }^{16}$ observed $86 \%$ of Vancomycin sensitive strains. It was also observed that, commonest Gram negative organism isolated was Klebsiella pneumoniae which was $91.3 \%$ susceptible to Meropenem, $84.05 \%$ to Amikacin and 56.52\% Ciprofloxacin. Most of other antibiotics were resistant. In view of the changing spectrum of the causative agents of neonatal septicemia and their antibiotic susceptibility patterns from time to time and from one hospital to another, a positive blood culture and the antibiotic susceptibility testing of the isolates are the best guide to the antimicrobial therapy. Blood culture is still the "Gold standard" for the diagnosis of septicemia in neonates and should be done in all cases of suspected septicemia prior to starting the antibiotics.

\section{Acknowledgement: NONE}

Conflict of Interest: The authors declare that they have no conflict of interest.

\section{References}

1. Agnihotri N, Kaistha N, Gupta V. Antimicrobial susceptibility of isolates from neonatal septicemia. Jpn J Infect Dis, 57(6), 2004, 273-5.

2. Tsering DC, Chanchal L, Pal R, Kar S. Bacteriological profile of septicemia and the risk factors in neonates and infants in Sikkim. Journal of global infectious diseases, 3(1), 2011, 42.

3. Chacko B, Sohi I. Early onset neonatal sepsis. The Indian Journal of Pediatrics, 72(1), 2005, 23-6.

4. Aggarwal R, Sarkar N, Deorari AK, Paul VK. Sepsis in the newborn. Indian $J$ Pediatr, 68, 2001, 1143-7

5. Shrestha S, Shrestha NC, Singh SD, Shrestha RP, Kayestha S, Shrestha M, Thakur NK. Bacterial isolates and its antibiotic susceptibility pattern in NICU. Kathmandu University Medical Journal, 11(1), 2014, 66-70.

6. Roy I, Jain A, Kumar M, Agarwal SK. Bacteriology of neonatal septicaemia in a tertiary care hospital of northern India. Indian Journal of Medical Microbiology, 20(3), 2002, 156.

7. Simonsen KA, Anderson-Berry AL, Delair SF, Davies HD. Early-onset neonatal sepsis. Clinical Microbiology Reviews, 27(1), 2014, 21-47.

8. Gyawali N, Sanjana RK. Bacteriological profile and antibiogram of neonatal septicemia. The Indian Journal of Pediatrics, 80(5), 2013, 371-4.

9. Jyothi P, Basavaraj MC, Basavaraj PV. Bacteriological profile of neonatal septicemia and antibiotic susceptibility pattern of the isolates. Journal of Natural Science, Biology and Medicine, 
4(2), 2013, 306.

10. Collee JG, Fraser AG, Marmion BP, Simmons A. Mackie and McCartney Practical Medical Microbiology. 14th ed. Edinburg, Churchill Livingstone, 1996.

11. Betty A. Forbes, Daniel F. Sahm, Alice S. Weissfeld. Bailey \& Scott's Diagnostic Microbiology. 11th ed. Mosby publications, Elsevier, 2002.

12. Gotoff SP, Behrman RE. Neonatal septicemia. The Journal of Pediatrics, 76(1), 1970, 142-53.

13. Roy I, Jain A, Kumar M, Agarwal SK. Bacteriology of neonatal septicaemia in a tertiary care hospital of northern India. Indian Journal of Medical Microbiology, 20(3), 2002, 156.

14. Bhat R, Lewis LE, Vandana KE. Bacterial isolates of early-onset neonatal sepsis and their antibiotic susceptibility pattern between 1998 and 2004, an audit from a center in India. Italian Journal of Pediatrics, 37(1), 2011, 1.

15. Manroe BL, Weinberg AG, Rosenfeld $\mathrm{CR}$, Browne R. The neonatal blood count in health and disease. I. Reference values for neutrophilic cells. The Journal of Pediatrics, 95(1), 1979, 8998.

16. Kayange N, Kamugisha E, Mwizamholya DL, Jeremiah S, Mshana SE. Predictors of positive blood culture and deaths among neonates with suspected neonatal sepsis in a tertiary hospital, Mwanza-Tanzania. BMC Pediatrics, 10(1), 2010, 1

17. Matthew J. Bizzarro, Veronika Shabanova, Robert S. Baltimore, Louise-Marie Dembry, The Rise and Fall of Coagulase-Negative Staphylococci Neonatal Sepsis 20042013: The journal of paediatrics, May 2015 Volume 166, Issue 5, Pages 11931199.

18. Eric Giannoni, MD, Philipp K.A. Agyeman, MD et all Neonatal Sepsis of Early Onset, and Hospital-Acquired and Community-Acquired Late Onset: A Prospective Population-Based Cohort Study for the Swiss Pediatric Sepsis Study, The journal of paediatrics, October 2018 Volume 201, Pp. 106114.

\section{How to cite this article:}

Ravindar Sirikonda and Kondal Rao Ravilla. 2018. Bacteriological Study of Neonatal Septicemia and their Antibiotic Susceptibility Patterns at MGM Hospital, Warangal, India. Int.J.Curr.Microbiol.App.Sci. 7(12): 1515-1522. doi: https://doi.org/10.20546/ijcmas.2018.712.179 